\title{
Using Multi-level Features Construction for Discovering Key Twitterers
}

\author{
Jianjun $\mathrm{Wu}$ \\ Department of Computer, Beijing College of Politics and Law, Beijing 102628, China \\ zhengfazuoye@163.com
}

Keywords: Social Networks, key users, Networks Analysis, Information Diffusion.

\begin{abstract}
Identifying key users have become a focal problem in the area of online social networks. Tweets and Behaviors of people are two core facets for finding key users. Tweets of published by users spread support from different behaviors. However, existing literature on key users evaluation has mainly focused on methods based on one of them effects in social network, which make topic and behavior latent dimensions to difficult to interpret. How to modeling roles of user revealing the hidden relation based on tweets that users interested in and behaviors for dissemination of information in real social networks? In this paper, we tackle this problem by focusing on different behaviors of users and tweets similarity based on word embedding to measure the influence of users in social networks. We propose an algorithm using relation feature construction for key twitters extraction. Through extensive experiments comparing with different algorithms, we demonstrate that model is able to identify key users. Additional, the model that can be used to facilitate other tasks such as automated latent community discovery, and to track origin users.
\end{abstract}

\section{Introduction}

Social Networks have been an important real-time information medium that has attracted users to express their opinions, publish tweets, following hot topics and influential. Many researchers in different domains are using network media (e.g,twitter, facebook, plurk.) to study network diffusion, trace emotional contagion and identify influence patterns. Through these channels ${ }^{[2]}$, users can interact, engage and share information with each other. The information ${ }^{[1]}$ has certain degree influence on user's choices, but the way and degree of its effect is not same. For example, People often refer to the opinions of their friends or general commercials when confronted with various decisions on consumer purchasing or online shopping process. By targeting the behaviors from a social network set of individuals, marketers possibly achieve very small marketing ${ }^{c o s t}{ }^{[3]}$.This is because individuals, a small minority of influential play the part of intermediaries between ordinary users and information source. However, social network have a large number of users and enormous amounts of data. It's a challenge of task for quantitatively assess ranks to identify the most influential users. Furthermore, the influential relationship strength is not easily formalized dynamics of interaction activity. Commonly, the diffusion path of information is difficult observed. We only extract propagation graph, disordered users that are affected by sharing similar information or topics in social network. Tang ${ }^{[4]}$ tackles this issue by using which individual has highest probability to affect other individuals. What is inherent to influence in social networks? The question is of great important that it extracts influential and measures their affecting scope. For instance, can we discover influential leaders such that come from the same group?

Traditional methods of influential extraction play a vital role based on link between the users. These methods produce influence nodes extracted from the explicit links path without referring to mostly the interchange of information among users. The essential assumption is that closer distance is more impact in social network. Furthermore, the emergence of information possibly have different sources, such as newspaper, radio stations, TV stations and online social media sites. In practice, it was hard to clear distinguish between what is closer from social networks and what is farther from external sources, mass media. Influential figures extraction based only on topology links can result in influential individuals which are interest in widely diverged, believe in different things or differ the scope of their followers. Thus, it is possible to have influence of different spanned distinct subjects. 
If figures come by the methods based on discussed information respectively which are similar. It's more fit the reality based senmatic interpretation. What is the grand essential of influence? Has Figure the same impact on the others' actions (such as retweet, comments, et.) cross different domains?

These challenges, as we have seen above, would still be possible to be solved for finding influential leaders in social networks based on the following aspects:

(1) Role reconstruction: In addition the connectivity relation, what is the most important role in influential recognized based on other traits in social networks?

(2)Influential gap: how to use effective strategies to break through the limitation of topic-specific relationship networks and bridge the gap between topic-based and link-oriented measuring the influence?

In response to these issues, we put forward to identify key player through comprehensive approaches by reconfigured role of users participated in discussed message and accumulated (positive and negative) influence across a broad of domains.

Based on these mentioned, we proposed multiple-level features to model the user-tweet level propagation influence on social network. It can efficiently find and identify key influencers from which communities are. The main contributions of this paper are summarized as follows:

(1) We propose new functions for measuring the Topic-Behavior level influence in social networks

(2) We provide a users-tweets Flow framework for individual actors influence analysis.

(3) We identify the key influential, as well as their played what role do.

The remainder of the paper is organized as follows. Problem statements and definitions are given in section 2. Section 3 introduce multiple-level model and user-tweet Flow framework. Section 4 evaluates the effectiveness of our algorithm comparing other algorithms. Section 5 listed related work. The last section summarizes our conclusion and future work.

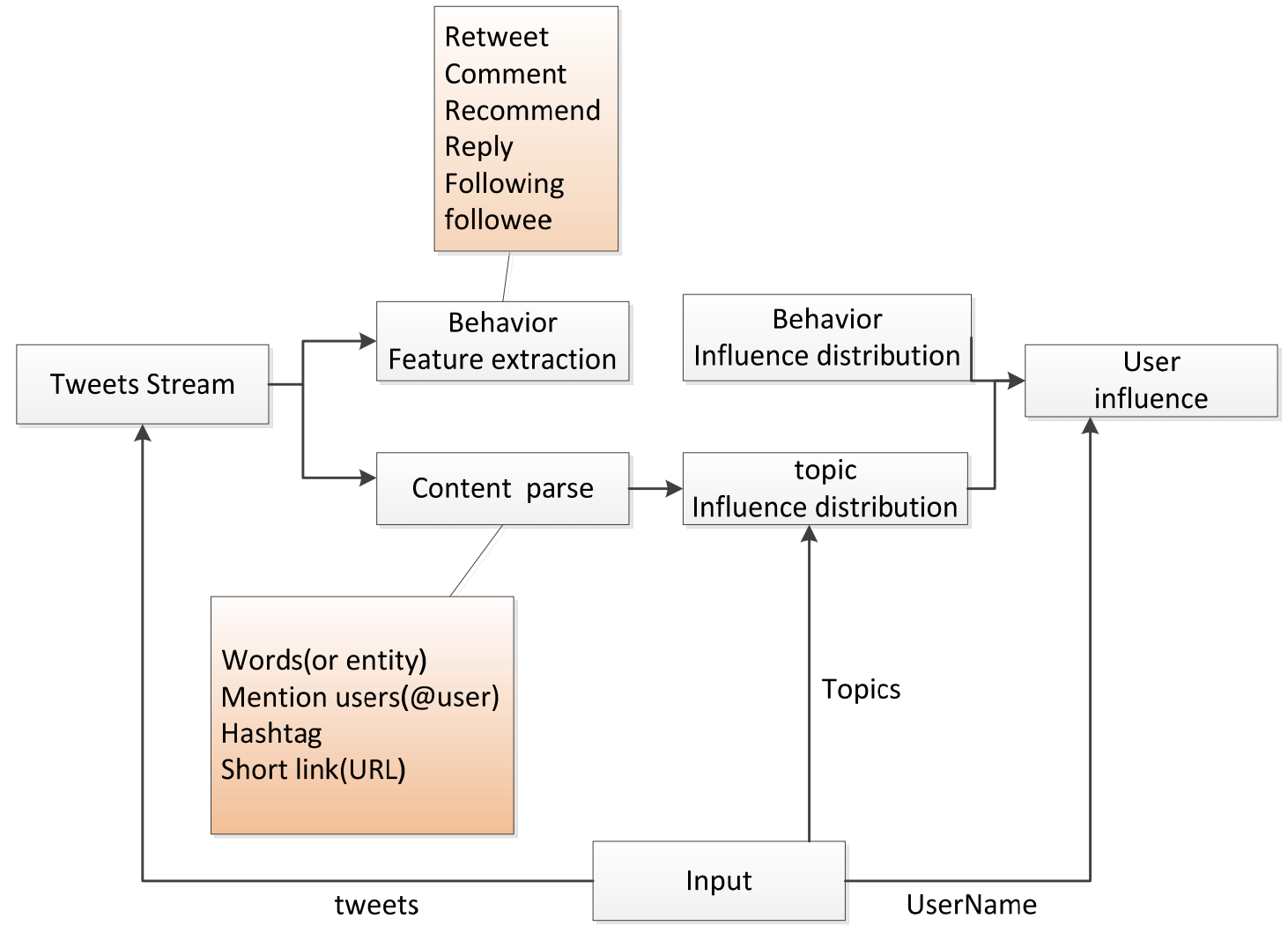

Figure 1: overview of tweets processing framework 


\section{Problem Formulation}

Suppose the hidden networks formed from connected by users' communitied behaviors, the latent senmatic networks formulated from users discussed relative topics in a social networks. The many methods evaluate users influence by modeling retweeting behaviors or cascades spreading, but not focusing on the role of propagate over an specific subject and for each of them effecting scope. The specific goal is to infer roles of users from the virtual network over mentioned relative information. Figure 1 describes input tweets stream processing mechanism. First many features are generated by input tweets stream parsed. We also leverage the social functionality to match the different features, and acquire influence distribution by viture of history data. The key aspects are feature extraction from tweets and behavior quantization.

\subsection{Problem Statement}

We represent a social network as undirected weighted graph $\mathcal{G}=(V, E, W)$. Given $V=\left\{v_{1}, v_{2} \cdots, v_{n}\right\}$ is the nodes set where $v_{i}$ denotes a social user, $\mathrm{E}$ is the edge set as well as a matrix $E_{j, z}^{i}=$ $\left\{\left\{e_{11}, e_{12}, \cdots, e_{m n}\right\}|i \in(m, r, f, p)| j=b ; i \in(h, t) \mid j=s\right\}$, Edge forms from interaction relationship between users directed from different behavior of users or tweets generated by users. Retweet of users on tweets will formulate edge set $E^{r}$ subnetwork from the same origin of tweets , $E^{f}$ edge set from following/followee relationship, $E^{m}$ that is mentioned users set from parsing of tweet content that reflect information will be automatically sent to receivers timeline by push. W is illustrative of the importment degree from users interaction with others. In contrast, $E^{p}$ presents relationship from users' tweets replied. In addition, the implicit relationship of senmatic analysis formed from hashtag and topical similarity. Different behavior of users formulate different subgraph through tweets spreaded. Given a tweet generated from origin user will be adopted or retweet through individual users that are compose of Retweet network.

Table1: Notation

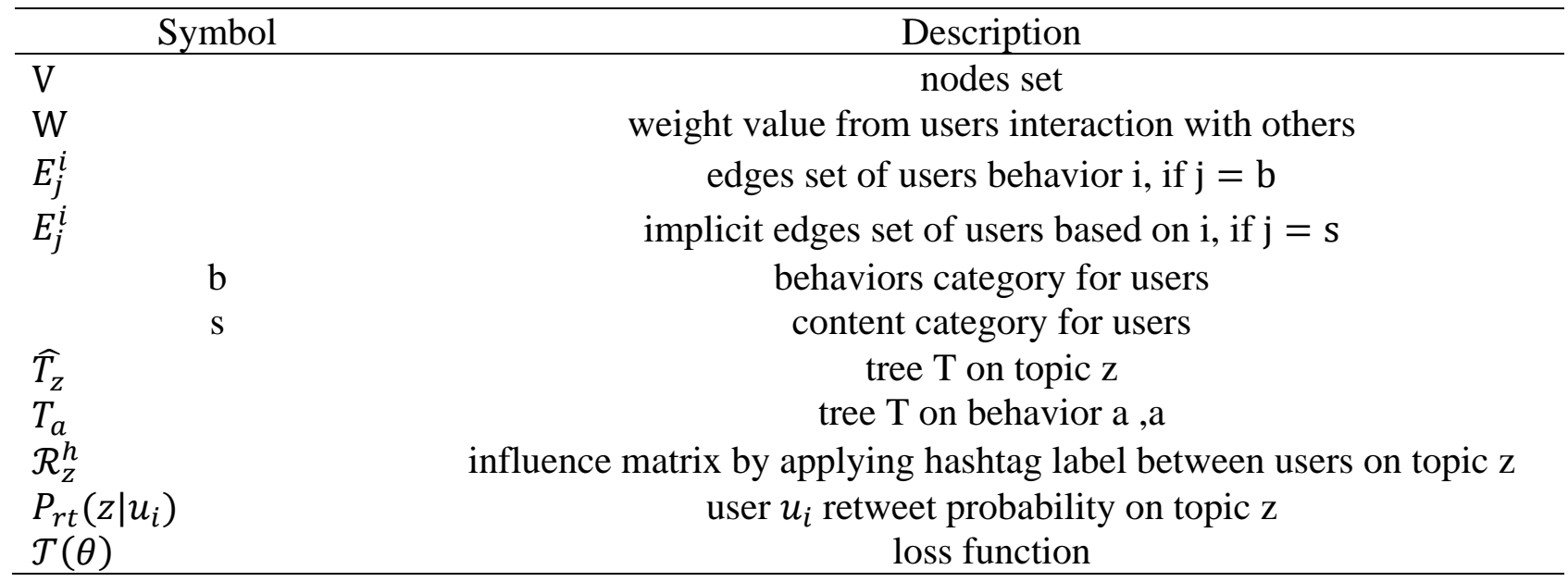

As mentioned in Section 1, users discussed the same topics would form implicit influence communities. Other users outside communities will not affect the communities even if they link from each other. This is also our work critical different point with other algorithms. The reason for not considering those users lies in its behaviors without participating in discussion the same topic.

\section{Model Formulation}

As described above, we present key twitterers extraction from three dimensions based on our framework: users' topic influence distributions from hashtag and word embedding similarity between users, behaviors influence distributions subgraph that come from different interaction activities among users, influence communities based on similar topics generated by different users.

\subsection{Latent Topic Model}

As stated in the mentioned, a user influenced other users by performing an preference tweets from others. As for users, all activities depend on tweets. It is the most important factors are needed to take into consideration. A tweet usually includes text, \# (hashtag), short link (url) and @user (mentioned 
user). Currently, the problem of data sparsity from the tweet in social network had been defined by its 140-character limit. We propose a short text feature extraction method that performs feature extraction on a short text based on hashtag and word embedding analysis method. A hashtag is a type of label or metadata tag used on social network which makes it easier for users to find messages with a specific topic or content. Hence, similar hashtag from different tweets have been discussed by different users will be concerned about the same topic. Similarly, these words from the similar context in tweets have most probabilistically to mean the same thing. We use word embedding method describing tweets and extract latent topic communities from similar words representation.

We define the LT model in a particular influence tree pattern T. Our goal is to obtain $P(T \mid u)$, the probability that a influence tree $\mathrm{T}$ creates from user $\mathrm{u}$. The tree is not consider time sequence defined only by topic similarity. We extract $\mathrm{T}$ by considering all possible influence subtree

$$
P\left(T_{Z} \mid u\right)=\sum_{T_{Z} \in \cup \Gamma(z)} P\left(T_{Z} \mid z\right) P(z \mid u)
$$

where $\mathrm{z}$ is the set of all topics and $\Gamma(z)$ is the set of all the undirected subtree on $\mathrm{z}$. The topics assume conditional independent given the user $u$. we don't observe particular subtree pattern generated by topical propagated. The tree $T_{Z}$ comes from merged subtree by topic similarity between users.we compute the probability of influence tree $T_{Z}$ on user u under the set of topics $\mathrm{z}$.

The latent topic model applies variables that includes explicit variables $\left\{u_{i} \mid i \in(1, \cdots, n)\right\}$ which corresponds to the users, $\left\{I_{u} \mid u \in(1, \cdots, n)\right\}$ as the set of tweets from user u published and implicit variables $\left\{e_{i j}^{z} \mid i \in(1, \cdots, n), j \in(1, \cdots, n)\right\}$ where present user $\mathrm{i}$ that has weight value e to influence user $\mathrm{j}$ on topic $\mathrm{z}$.

In figure 2,The example shows a process flow about LT. Different subnetworks have been created by extracted hashtag and computed word embedding under tweets from given users have been parsed. The edges $\left\{E_{j, Z}^{i} \mid j \in s, i \in(h, t)\right\}$ between users indicate two social relationships extracted from original tweets.

Similarly, we cluster the hashtags released from a user together a hashtag set, aggregate the tweets published by the same user forming a paragraph set $I_{u}$

$\mathcal{R}_{z}^{h}$ is a user influence matrix by applying hashtag label between users on topic $\mathrm{z}, \mathcal{R}_{z}^{s}$ matrix presents user influence by applying word embedding similarity between $I_{u}$, The $P\left(T_{z} \mid z\right)$ is a probability users set from the dot product between $\mathcal{R}_{z}^{h}$ and $\mathcal{R}_{z}^{s}$

$$
P\left(T_{Z} \mid z\right)=\underset{T_{Z} \leq n}{\operatorname{argmax}}\left[\sum_{u_{i=1}^{k}}^{k} \mathcal{R}_{Z}^{h} \odot \mathcal{R}_{z}^{s} / \sum_{u_{i=1}}^{n} \mathcal{R}_{Z}^{h} \odot \mathcal{R}_{Z}^{s}\right]
$$

where $\mathrm{k}$ is not more than $\mathrm{n}$.

$P(z \mid u)$ describes a probability from user $\mathrm{u}$ interest on topic $\mathrm{z}$ in using LDA ,tweets from user $\mathrm{u}$ corresponds to $I_{u}$.

$$
P\left(T_{Z} \mid u\right)=\sum_{T_{Z} \in \cup \Gamma(z)} \underset{T_{Z} \leq n}{\operatorname{argmax}}\left[\sum_{u_{i=1}^{k}}^{k} \mathcal{R}_{z}^{h} \odot \mathcal{R}_{z}^{s} / \sum_{u_{i=1}^{n}}^{n} \mathcal{R}_{z}^{h} \odot \mathcal{R}_{Z}^{s}\right] P(z \mid u)
$$

\subsection{Behavior Transmission Model}

To deal with the influence problem that vary in frequency and intensity, we

present the behavior transmission model for formulating user behaviors. In this paper, we focus on studying the effects of four behaviors: retweet, reply, mention,

following /followee. We extract behavior tree $\mathrm{T}$ by considering all possible influence subtree

$P\left(T_{a} \mid u\right)=\sum_{T_{a} \in \cup \Gamma(z)} P\left(T_{a} \mid z\right) P(z \mid u)$

where a is the set of all behaviors and $\Gamma(z)$ is the set of all the undirected

subtree on z. Most of works on user behaviors influence puts particular emphasis on retweet and following/followee because their existing between directly connected users relationships, especially in the retweet behaviors meaning really influence occurring between users, others are not. To quantify this, we measure the strength of relatedness between users in a subnetworks and use the method of the multi-features fusion to obtain influence networks of users' behaviors.

Given an topic z, the behaviors of user u adoption includes retweet, reply, mention, and influence. According to user behavior history, we calculate the percentage of behavior accompanied tweet adopted by user u on topic z. 


$$
P_{r t}\left(z \mid u_{i}\right)=\frac{1}{\left|B_{(i, u)}\right|} \frac{\sum_{a=1}^{k} r t_{a}^{u_{i}}}{\sum_{u_{i}}^{u_{n}} \sum_{a=1}^{k} r t_{a}^{u_{i}}}
$$

Here we define the influence of the user $u_{i}$ on topic $\mathrm{z}$ is evaluated by selecting maximum value from three behaviors influence as:

$$
P\left(z \mid u_{i}\right)=\max \left\{P_{r t}\left(z \mid u_{i}\right), P_{r p}\left(z \mid u_{i}\right), P_{m}\left(z \mid u_{i}\right)\right\}
$$

As for the tree $T_{a}$ on topic $\mathrm{z}$, we also define probability function by searching the longest path tree from following/followee relationship as:

$$
P\left(T_{a} \mid z\right)=\max \left\{P\left(T_{r t} \mid z\right), P\left(T_{r p} \mid z\right), P\left(T_{m} \mid z\right)\right\}
$$

where $T_{r t}$ reflects retweet path tree by searching for following/followee relationship

set, $T_{r p}$ denotes reply action by user and $T_{m}$ means mentioned by user as the same.

The social behaviors influence tree $\mathrm{T}$ given user $\mathrm{u}$ can be described is then

$$
P\left(T_{a} \mid u\right)=\max \left\{P\left(T_{r t} \mid z\right), P\left(T_{r p} \mid z\right), P\left(T_{m} \mid z\right)\right\} * \max \left\{P_{r t}\left(z \mid u_{i}\right), P_{r p}\left(z \mid u_{i}\right), P_{m}\left(z \mid u_{i}\right)\right\}
$$

where we are applying for one of behaviors in the topic $\mathrm{z}$ by no means merged subtree of every action on tweets.

\subsection{Influence-maximizing Aggregation}

In this section, we present influence comprehensive method by mentioned above topic influence tree and behavior influence tree, which evaluate user influence in single topic $z$. The influence tree of a user on topic $\mathrm{z}$ can be merged to measure user influence on topic $\mathrm{z}$, which is calculated as:

$$
\widehat{T}_{Z}=P_{Z}(T \mid u)=P\left(T_{a} \mid u\right) P\left(T_{Z} \mid u\right) \propto \underset{t=z}{\operatorname{argmax}}\left\{\frac{\left(T_{a} \cap T_{Z}\right) \cup\left|T_{a}-T_{Z}\right|}{T_{a} \cup T_{Z}}\right\} \doteq \quad \underset{t=z}{\operatorname{argmax}}\left\{\left(T_{a} \cap T_{Z}\right) \cup\left|T_{a}-T_{Z}\right|\right\}
$$

Next, we define the user global influence can be measure as an aggregation of single-topic as:

$\widehat{T}=P(T \mid u)=\sum_{z}^{n} \widehat{T}_{z}+b_{z}$

where $\widehat{T}_{z}$ is the influence tree for topic $\mathrm{z}$ and $b_{z}$ is a bias to topic $\mathrm{z}$.

In order to solve optimization problem, we find $\hat{T}$ that solves the optimization problem by minimize loss function of influence tree is then

$\mathcal{T}(\theta)=\sum_{s=1}^{S} \widehat{T}_{(u, \theta)}\left[\Delta\left(T, T^{s}\right)\right]$

where $\widehat{T}_{(u, \theta)}$ is influence tree on user $\mathrm{u}, \theta$ is a hidden virable from topics.

The optimization problem are turned into find minimize deviation between influence trees. then

$$
\hat{\theta}_{M L}=\operatorname{argmin}_{\theta}\{\mathcal{T}(\theta)\}
$$

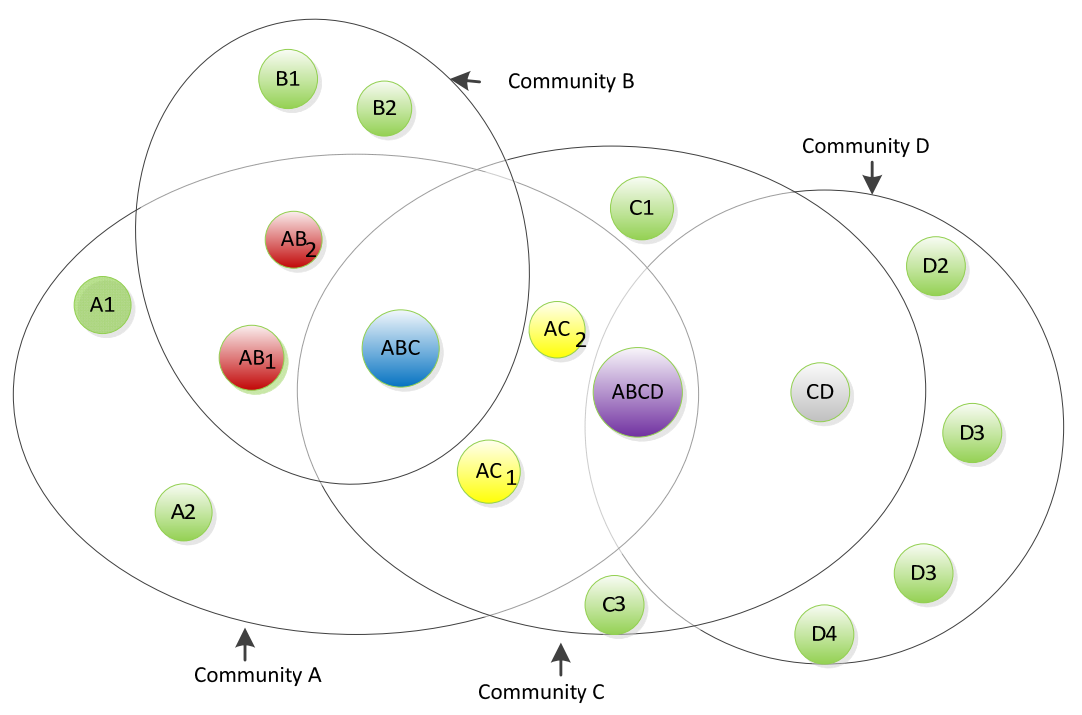

Figure 2: Influence diffusion problem from different (A, B, C, D) communities. Nodes represent bloggers (or social users), the relationship between bloggers (or users) express connected by means

\subsection{Discovering Community Influentials} of interbehaviors, similar interesting.

The aforementioned model presents a feasible way of identifying influential, but the influence of users are vary in different topics. In other words, user A on topic z is the most influential, not sure for 
topic c. In Figure 2, For example user $A B_{1}$ and user $A B_{2}$ from community B have bigger power, not influence in community $C$. Moreover, user $A B C$ has more widespread influence owing to it across different community of latent topic. It is a topical organizer and initiator. The Approach mentioned in 3.2 and 3.3 section can ascertain the effects scope of user, but we don't know the rank of influence on special topic. We consider user role as one of the parameters that may affect influence of the user. Although there are many factors used measurement that quantify the rank of users, for the sake of simiplicity, we use user participating in the number of topics and play the role of topics propagated as s heuristic measure of range affects of users in the community. We define a rank function of user, which uses linear combination of both features represents the role of influence a user given special topics. By the means of Equation $\mathrm{x}$, we get the following fully defined rank of influence model in community:

$\mathrm{R}(\mathrm{u})=\sum_{c} \omega\left(T_{Z}\right) P\left(T_{Z} \mid u\right)+a_{u}+S_{u}$

where Community $\mathrm{C}$ is latent community of topic $\mathrm{z}, \omega$ is a weight function,which rewards or penalizes the influence score of $\mathrm{u}$ according to the length $T_{Z}$ of user. $S_{u}$ is a bias to denote the role of user $\mathrm{u}$ initiating tweets. $a_{u}$ for organize role on topics.

\section{Experimental Evaluation}

In this section, we first discuss the need for experimental data, perform our various experiments on two real-world data sets: Unofficial Apple Weblog and Twitter data, and evaluate the efficiency of our approach on the two data sets. We also compare the MIIB, TwitterRank with our approach.

\subsubsection{Data Sets and Experiment Settings}

TUAW data freely available for research from [MIIB], having blog posts of five years from 2004 to 2008. It has been used by comparing influential with our approach But TUAW data is no available bloggers' interacting information for the purposes of our experiments on user's behavior. Hence, we used in this study was crawled social data from twitter.com that provides some traits, like followee/following,@ ation,retweet behaviors for blogger identification. We crawled one post which contain the number of favorite, retweet, @ users, \# hashtag, date and time of posting.

\subsubsection{Evaluation Criteria}

For quantitatively evaluate effectiveness of our method, we compare TR to the following related baseline models: MIIB. This is a model of identify top influential users by TUAW dataset.

Twitter Rank. It is an extension of PageRank algorithm, measures user influence based on topical similarity between users and link structure based on Twitter dataset.

Feature-based. We use different features to obtain the list of top ten users quantitating influence of features.

Community-based. TR allows us to automatically discover users influence scope based on senmatic similarity of post.

\subsection{Results and Discussions}

Now we evaluate the effectiveness of our algorithm in the following section on TUAW and Twitter data sets.

\subsubsection{Topic Analysis}

Results in terms of senmantic similarity from TUAW data sets are shown in Figure 3. We observe comparatively dispersibility topics under the same blogger based on senmantic similarity of posts and posts title. The blogger has focusing on the more topics, the better activieness. The less words senmantic similarity compare, the more drift of the topics. But posts have strong correlation title under the same blogger. It shows the comparison between posts and titles changing trends that are nearly the same. 


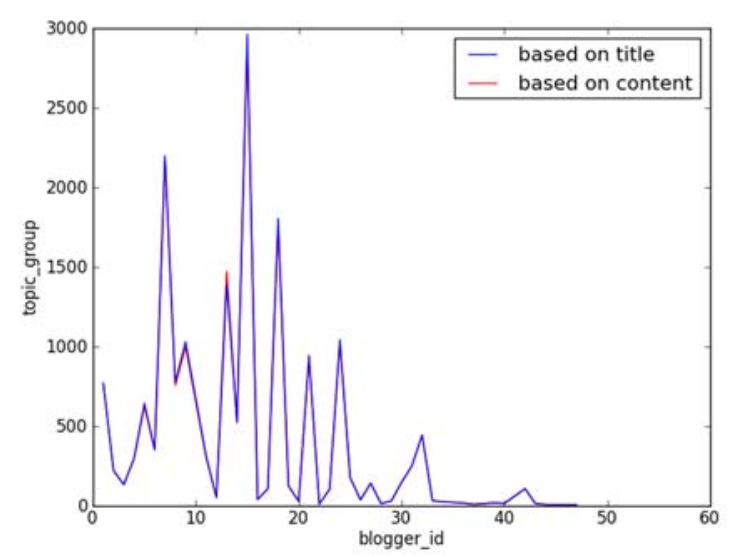

Figure 3: Topic similarity based on title and content from one user

It can be seen from the table2, the top influential users based on single feature from topic and title are nearly the same. Those indicate the theme is a significant feature, and with the same effect the content. The titles show a good indicated from posts. The comments and inlinks are not good as topic in evaluation users influence stability. It is interesting to notice that the results top one user Scott McNulty produce the same from TR and MIIB. We can note that Scott McNulty has been ranked one in different features including topic and title, but we find Scott McNulty published 3037 post from TUAW data sets that shows its high activeness on the topics. Similarly, C.K. S, III has been more active on topics ranked at top position although its has more variations in the ranks by other features.

Table 2: Top10 influential users using single feature

\begin{tabular}{ccccc}
\hline rank & M-comments & M-inlink & Topic-multiformity & Title-similarity \\
\hline 1 & Scott McNulty & Cory Bohon & Scott McNulty & Scott McNulty \\
\hline 2 & Erica Sadun & Erica Sadun & Dave Caolo & Dave Caolo \\
\hline 3 & Dave Caolo & Robert Palmer & David Chartier & David Chartier \\
\hline 4 & David Chartier & Dave Caolo & Erica Sadun & Erica Sadun \\
\hline 5 & Victor Agreda, Jr. & Mike Schramm & C.K. Sample, III & C.K. Sample, III \\
\hline 6 & Mat Lu & Michael Rose & Mat Lu & Mat Lu \\
\hline 7 & Cory Bohon & Mat Lu & Laurie A. Duncan & Laurie A. Duncan \\
\hline 8 & Michael Rose & Steven Sande & Cory Bohon & Michael Rose \\
\hline 9 & Mike Schramm & Scott McNulty & Michael Rose & Cory Bohon \\
\hline 10 & Robert Palmer & Brett Terpstra & Mike Schramm & Mike Schramm \\
\hline
\end{tabular}

Table 3: Top10 influential users based on different algorithms discovering from TUAW

\begin{tabular}{|c|c|c|c|c|}
\hline rank & MIIB & MIBIX & MIBI & TBRank \\
\hline 1 & Scott McNulty & Cory Bohon & Cory Bohon & Scott McNulty \\
\hline 2 & Erica Sadun & Robert Palmer & Robert Palmer & Dave Caolo \\
\hline 3 & Dave Caolo & Steven Sande & Steven Sande & C.K. Sample, III \\
\hline 4 & David Chartier & Erica Sadun & Erica Sadun & Mat Lu \\
\hline 5 & Cory Bohon & Christina Warren & Michael Rose & Sean Bonner \\
\hline 6 & Victor Agreda,Jr. & Michael Rose & Mike Schramn & Mike Schramm \\
\hline 7 & Mat Lu & Mike Schramn & Christina Warren & Erica Sadun \\
\hline 8 & Michael Rose & Mat Lu & Dave Caolo & Michael Rose \\
\hline 9 & Mike Schramn & Dave Caolo & Mat Lu & $\begin{array}{c}\text { Victor Agreda, } \\
\text { Jr. }\end{array}$ \\
\hline 10 & Robert Palmer & Brett Terpstra & Brett Terpstra & David Chartier \\
\hline
\end{tabular}

Using single feature and different algorithms, we compare the correlation between the top 10 rank lists. Pearson rank correlation coefficient, which takes value from 1 to -1 ,was used to measure the two rank lists whether or not are related, if $\mathrm{p}=1$, the two list are nearly the same, and $\mathrm{p}=-1$ if one rank lists is reverse of the other. It is used to measure the level of agreement between users rank lists given 
by TR and the other algorithms. As can be seen from Table 4(a), 4(b) and 4(c), the results show the users rank lists computed by the TR is strong correlated with the users rank lists extracted by TwitterRank in twitter dataset more than MIIB computed from tuaw dataset.

Table 4: Correlation with different algorithms by Top10 Rank

(a) Single Feature Rank

\begin{tabular}{|c|c|}
\hline & Kendall \\
\hline M_comments vs. Topic_m & 0.6 \\
\hline M_comments vs. M_link & 0.2 \\
\hline
\end{tabular}

(b) Correlation with different algorithms by Top10 Rank

\begin{tabular}{|c|c|}
\hline & Kendall \\
\hline TR vs. MIIB & 0.6444444444 \\
\hline TR vs. MIBIX & 0.0222222222 \\
\hline TR vs. MIBI & 0.1111111111 \\
\hline MIBIX vs MIB & 0.0666666667 \\
\hline
\end{tabular}

(c) Correlation with TwitterRank

\begin{tabular}{|c|c|c|}
\hline & & Kendall \\
\hline Rank 10 & TR vs. TwitterRank & 0.7777777778 \\
\hline Rank 20 & TR vs. TwitterRank & 0.9473684211 \\
\hline
\end{tabular}

\section{Related Work}

Key influentials discovered from online social network have been extensively research in different domains. Previous studies also focus on user relationship based on link information of following/followee using twitter features. Some algorithms, such as KHYRank, IARank ${ }^{[5,6,7]}$,RageRank-like, ranking users influence primary focus on the information spreaded link path of users,. Similarly, TwitterRank uses link structure compromiseing topical similarity to measure the influence but [12] utilizes a priori information to measure topic-sensitive influence. TunkRank is a analog of PageRank method, defines the influence of twitter from number of its published tweets from followers read ${ }^{[8]}$. Imen ${ }^{[9]}$ et al. present a supervised learning classification model to identify the prominent users under the specific topic. Manuel ${ }^{[10]}$ proposes NETINF algorithm which defines cascade propagation model to infer implicit networks of influence and diffusion. Tang ${ }^{[4]}$ analyzed topic-level social influence by proposing TAP method to apply large scale networks. Likewise, [11] propose methods to qualitatively measure item-level influence. As for as I know, existing work has not been implemented for quantitatively measuring the multiple-level influence in social networks, and ascertained influential role.

Our work presented builds on the gap between structural approaches and topic-based methods. we propose multiple-level model for quantitatively extracting and discovering key influentials from special latent community.

\section{Conclusion}

This paper addresses a novel method of extracting influential users in social netoworks. We propose a topic-behavior level approach to describe the problem of three dimensions: user, topic and behavior. Moreover, we propose tweets processing framework to assist the task to extract influential users. The experimental results show that the proposed topic-behavior level method is correlation with different metrics and more exactly portray influential users. In addition, we demonstrate that LT model outperforms other related algorithm in depict key leaders in different latent community on topic. What the most important thing is word embedding and hashtag that have introduced into our model by means of text representation to relief data sparsity problem. The influential users based on social influence draw a 360 all-round portrait would be an interesting future work. 


\section{References}

[2]. Milstein, S., Lorica, B., Magoulas, R., Hochmuth, G., Chowdhury, A., Tim: Twitter and the Micro-Messageing revolution: Communication, connections, and Immediacy-140 Characters at a time. O’Reilly Media, Incorporated (2008)

[3]. Nail, J.: The consumer advertising backlash. J. Forrester Research (2004)

[4]. Katz, E., Lazarsfeld P.F.: Personal influence, The part played by people in the flow of mass communications, The Free Press, New York (1955)

[5]. Tang J, Sun J, Wang C, et al. Social influence analysis in large-scale networks. In: 15th ACM SIGKDD International Conference on Knowledge Discovery and Data Mining, pp. 807-816. ACM, Paris (2009)

[6]. Cappelletti, R., Sastry, N.: IARank: Ranking Users on Twitter in Near Real-time, Based on their Information Amplication Potential. In: 4th International Conference on Social Informatics, pp. 70-77. IEEE, Lausanne (2012)

[7]. Weng, J.S.,Lim, E-P., el at: TwitterRank:Finding Topic-sensitive Influential Twitterers. In: 3th ACM International Conference on Web Search and Data Mining, pp. 216-231. ACM, New York (2010)

[8]. Yu, A., Hu, C., V, Kilzer, A.: KHYRank: Using Retweets and Mentions to Predict Influential Users (2011)

[9]. A twitter analog to pagerank, http://thenoisychannel.com/2009/01/13/a-twitter-analog-to-pagerank/

[10]. Bizid, I., Nayef, N., Boursier, P., Faiz, S., Morcos, J.: Prominent Users Detection during Specific Events by learning on- and off-topic Features of User activities. In: 2015 IEEE/ACM International Conference on Advances in Social Networks Analysis and Mining, pp. 500-503. ACM, New York (2015)

[11]. Gomez-Rodriguez M, Leskovec J, Krause A. Inferring Networks of diffusion and influence. In: 16th ACM SIGKDD International Conference on Knowledge Discovery and Data Mining, pp. 1019-1028. ACM, New York (2010)

[12]. Cui, P., Wang, F., Liu, S.W., el at: who should share what? Item-level social influence prediction for users and posts ranking. In: the 34th international ACM SIGIR conference on Research and development in Information Retrieval, pp. 185-194. ACM, New York (2011)

[13]. Katsimpars, G., Vogiatzis, D., Paliouras, G.: Determining Influential Users with Supervised Random Walks. In: the 24th International Conference on World Wide Web, pp. 787-792. ACM, New York (2015) 